\title{
SERS Microspectroscopy of Biomolecules on Dried Ag Colloidal Drops
}

\author{
P. Šimáková, M. Procházka, and E. Kočišová \\ Institute of Physics, Faculty of Mathematics and Physics, Charles University, \\ Ke Karlovu 5, 12116 Prague 2, Czech Republic
}

Correspondence should be addressed to P. Šimáková, simakova@karlov.mff.cuni.cz

Copyright (C) 2012 P. Šimáková et al. This is an open access article distributed under the Creative Commons Attribution License, which permits unrestricted use, distribution, and reproduction in any medium, provided the original work is properly cited.

\begin{abstract}
We report the application of dried Ag hydroxylamine-reduced colloidal drops to surface-enhanced (resonance) Raman scattering (SE(R)RS) study of biomolecules using Raman microspectroscopy. 5,10,15,20-tetrakis(1-methyl-4pyridyl)porphyrin (TMPyP), amino acid tryptophan, and phospholipid 1,2-distearoyl-sn-glycero-3-phosphocholine (DSPC) served as testing biomolecules. Ag colloid/biomolecule drop dried on glass support forms a ring in the edge part of the drop in which almost all nanoparticles are clustered. This specific drying process promotes adsorption of the studied biomolecules in highly enhancing sites ("hot spots") as well as concentrates them in the ring. We were able to obtain SE(R)RS spectra from the ring that cannot be acquired directly from Ag colloidal solutions (SERRS spectrum of $1 \times 10^{-10} \mathrm{M}$ TMPyP by $1 \mathrm{~s}$ accumulation time, SERS spectrum of $2 \times 10^{-7} \mathrm{M}$ DSPC). Despite the spectral irreproducibility and problems with spurious bands in some cases, SERS microspectroscopy of studied biomolecules using dried Ag colloid/adsorbate systems improves SERS applicability and sensitivity in comparison to measurements directly from Ag colloidal solution.
\end{abstract}

Keywords: SERS, Ag colloidal nanoparticles, porphyrin, tryptophan, phosphatidylcholine

\section{Introduction}

Surface-enhanced Raman scattering (SERS) spectroscopy is a very useful detection and analytical technique thanks to the enhancement of Raman scattering for molecules adsorbed on rough metal surfaces $[1,2]$. The enhancement is usually up to $10^{6}$, but selective excitation of extremely highenhancing sites ("hot spots") can give SERS enhancement of the order of $10^{12}$ allowing even singlemolecule detection $[1,2]$.

Silver and gold colloidal suspensions prepared by chemical reduction still belong to popular SERS-active substrates because of their simple and cheap preparation and sufficient SERS enhancement. Drawbacks of colloidal solutions are their low spectral reproducibility due to the colloid aggregation which generally increases SERS enhancement, but is very difficult to control [2]. Moreover, the diffusion of aggregates in the solution causes signal fluctuations. More stable SERS substrates can be obtained by immobilization of colloidal nanoparticles on glass via silane $[3,4]$ or by drying $[2,4,5]$. In the latter case, during the evaporation of a colloidal or colloid/adsorbate drop on glass surface the "coffee ring effect" [6] causes formation of a ring at the contact line of the drop containing most of the nanoparticles. 
Aggregation of the nanoparticles upon drying can create fractal-like aggregates with "hot spots" [2]. One can expect that drying promotes adsorption of the studied compound in such highly enhancing sites as well as concentrates it in the ring. Although dried drops are neither uniform nor reproducible substrates [2], SERS measurement from the ring can bring some advantages in comparison to measurement directly from the colloidal solution.

In this contribution we report the application of dried Ag hydroxylamine-reduced colloidal drops to SE(R)RS study of biomolecules using Raman microspectrometer. We chose 5,10,15,20-tetrakis(1methyl-4-pyridyl)porphyrin (TMPyP), amino acid tryptophan, and phospholipid distearoylphosphatidylcholine (DSPC) as testing biomolecules.

\section{Experimental}

$\mathrm{AgNO}_{3}, \mathrm{NH}_{2} \mathrm{OH} \cdot \mathrm{HCl}, \mathrm{NaOH}, 5,10,15,20$-tetrakis(1-methyl-4-pyridyl)porphyrin (TMPyP) and Ltryptophan were purchased from Sigma-Aldrich and 1,2-distearoyl-sn-glycero-3-phosphocholine (DSPC) from Avanti Polar Lipids, Inc. Deionized water of a specific resistance of $18 \mathrm{M} \Omega \mathrm{cm}$ was used. Liposomes of $\sim 400 \mathrm{~nm}$ in diameter were prepared by extrusion of $1 \mathrm{mg} / \mathrm{mL}(1.3 \mathrm{mM})$ DSPC water suspension through a polycarbonate membrane filter of the LiposoFast-Basic apparatus (Avestin, Inc.) [7].

Ag colloid was prepared by reduction of $\mathrm{AgNO}_{3}$ by hydroxylamine hydrochloride [8] according to protocol used in [5]. Aqueous solution of the biomolecules was mixed with Ag colloid to obtain the desired concentration and $\sim 2 \mu \mathrm{L}$ of the colloid/biomolecule mixture was dropped by pipette on a clean glass slide (cleaned with diluted $\mathrm{HNO}_{3}$ and ethanol). The drop was left to dry at room temperature for approximately $45 \mathrm{~min}$.

SE(R)RS spectra were recorded by a confocal Raman microspectrometer LabRam HR800 (Horiba Jobin Yvon) equipped with a 600 grooves/mm grating and a nitrogen-cooled CCD detector. Objectives $(100 \mathrm{x}, \mathrm{NA}=0.9$ or $50 \mathrm{x}, \mathrm{NA}=0.7)$ provided $1-2 \mu \mathrm{m}$ laser spot on the sample. Excitation lines $514.5 \mathrm{~nm}$ of an $\mathrm{Ar}^{+}$laser (Melles Griot, power $\sim 0.19 \mathrm{~mW}$ at the sample) and $632.8 \mathrm{~nm}$ of an internal He-Ne laser $(\sim 0.07 \mathrm{~mW}$ at the sample) were used. Spectra were collected at room temperature $\left(20^{\circ} \mathrm{C}\right)$. Microscopic images of the dried drops were taken by the built-in digital camera using objectives $5 \mathrm{x}$ and 50x.

\section{Results and Discussion}

Ag colloid mixed with TMPyP, tryptophan, or DSPC dropped carefully on clean glass surface forms a ring in the edge part of the drop in which almost all nanoparticles are clustered. An example of such structure is shown in Figure 1 for the Ag hydroxylamine-reduced colloid with TMPyP $(1 \times$ $10^{-7} \mathrm{M}$ concentration). The ring consists of big aggregates of colloidal nanoparticles and crystals of $\mathrm{NaCl}$ coming from the colloid preparation. Formation of a clearly distinguishable ring was observed for various TMPyP concentrations, that is, for different aggregation states of the colloid, including strongly aggregated $\left(1 \times 10^{-6} \mathrm{M}\right)$ and nonaggregated $\left(1 \times 10^{-9} \mathrm{M}\right.$ and lower $) \mathrm{Ag}$ colloid/TMPyP systems, although the ring had different width for each particular TMPyP concentration. 


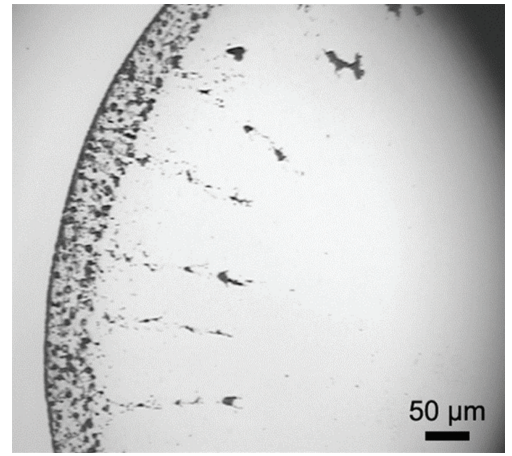

(a)

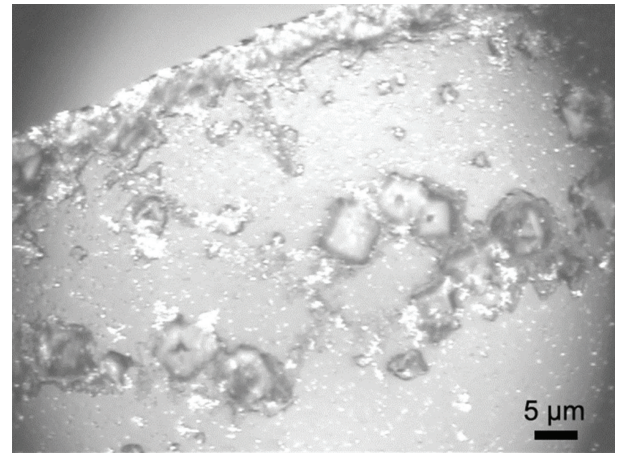

(b)

Figure 1: Optical microscope images of a dried Ag hydroxylamine-reduced colloid/TMPyP drop with $1 \times 10^{-7} \mathrm{M}$ TMPyP concentration: edge part of the drop (a) and detail of the ring (b).

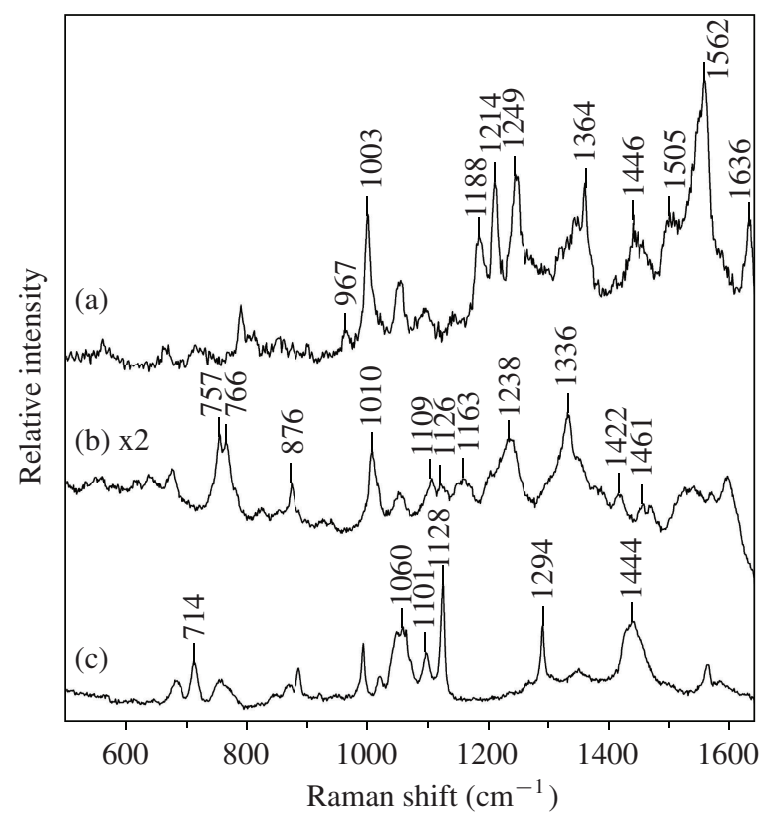

Figure 2: Examples of SE(R)RS spectra obtained from the dried Ag colloid/biomolecule drops with (a) $1 \times 10^{-7}$ M TMPyP (514.5-nm excitation, $1 \mathrm{~s}$ ), (b) $1 \times 10^{-5} \mathrm{M}$ tryptophan (632.8-nm excitation, $1 \mathrm{~s} \times$ $60)$ and (c) $2 \times 10^{-6} \mathrm{M}\left(1.7 \times 10^{-3} \mathrm{mg} / \mathrm{mL}\right)$ DSPC (632.8-nm excitation, $\left.5 \mathrm{~s} \times 40\right)$.

TMPyP is a suitable molecule for SERS because it provides high SERS signal owing to its structure and positive charge which helps adsorption on the Ag nanoparticles surrounded by negative ions. We measured the concentration dependence of SERRS spectra of TMPyP at $514.5 \mathrm{~nm}$ excitation in the concentration range $1 \times 10^{-6} \mathrm{M}-1 \times 10^{-10} \mathrm{M}$ and $1 \mathrm{~s}$ accumulation time (it is resonance Raman scattering because the excitation falls into an absorption Q-band of TMPyP). An example of the SERRS spectrum of $1 \times 10^{-8} \mathrm{M}$ TMPyP is demonstrated in Figure 2 (spectrum (a)). It shows signs of metalation 
(bands at $1364 \mathrm{~cm}^{-1}$ and $1562 \mathrm{~cm}^{-1}$ ), belonging to a metalation form in which $\operatorname{Ag}(0)$ atoms (probably induced by chlorides present in the colloid from the reduction agent) are incorporated into the porphyrin macrocycles [9]. Spectral features of TMPyP (marked in the spectrum (a) on Figure 2) were observed for all studied TMPyP concentrations (even for $1 \times 10^{-10} \mathrm{M}$ ) although some other bands not assigned to TMPyP can be seen. These spurious spectral bands (fluctuating narrow bands in the spectral region $1200-1700 \mathrm{~cm}^{-1}$ and/or two broad bands with maxima at $\sim 1350 \mathrm{~cm}^{-1}$ and $\sim 1580 \mathrm{~cm}^{-1}$ ) come from carbon species contamination and/or burning of the sample [4]. However, in the case of dried drops, problems with spurious bands were negligible compared to Ag nanoparticles immobilized by silane [4]. We have to emphasize that SERRS spectra of such low TMPyP concentrations $\left(1 \times 10^{-10} \mathrm{M}\right.$ detected even by $1 \mathrm{~s}$ accumulation time) cannot be measured directly from Ag colloidal solution.

SERS spectra of tryptophan obtained using Ag colloids have been reported in the literature recently [10-12], but the results significantly differ from each other. Such discrepancy can be explained by particular experimental conditions ( $\mathrm{pH}$, used $\mathrm{Ag}$ colloid and its activation, mode of adsorption, and consequently orientation of tryptophan on the surface, etc.). We measured SERS spectra of $1 \times 10^{-5} \mathrm{M}$ tryptophan at 632.8-nm excitation. An example is spectrum (b) in Figure 2. We have to note that our SERS spectra of tryptophan are completely different than that obtained by Aliaga et al. [10] from the same SERS system (dried hydroxylamine-reduced Ag colloid/tryptophan drop) but in good agreement with Raman spectra measured from Ag colloidal solutions [11, 12] and solid powder [12]. In Figure 2, spectrum (b), only spectral bands that can be surely assigned to tryptophan are marked [12]. We note that some spurious bands caused by burning of the sample might occur at certain spots and/or using higher laser power.

SERS studies of lipids using Ag colloidal solutions have not been reported up to now. Presence of both positive and negative charges on lipid heads makes adsorption of lipids on colloidal nanoparticles difficult. Moreover, long lipid chains could hinder nanoparticles aggregation. We were able to obtain SERS spectra of DSPC in $2 \times 10^{-6} \mathrm{M}$ and $2 \times 10^{-7} \mathrm{M}$ concentrations from dried Ag colloidal drops using $632.8 \mathrm{~nm}$ excitation. DSPC was prepared in the form of liposomes which are spherical lipid bilayers and represent a good model of biological membranes. An example of the SERS spectrum of DSPC in $2 \times 10^{-6} \mathrm{M}$ concentration with marked spectral bands of the phospholipid is shown in Figure 2 (spectrum (c)). Spectral features agree with those of normal Raman spectra of phosphatidylcholine $[7,13]$ although some spurious bands occurred. No SERS spectra of DSPC were obtained directly from Ag colloidal solution at the same concentration.

\section{Conclusions}

Ag hydroxylamine-reduced colloid/biomolecule drop dropped carefully on clean glass surface forms a ring in the edge part of the drop in which almost all nanoparticles are clustered. It seems that drying process promotes adsorption of some analytes (e.g. lipids) on Ag nanoparticles and "hot spots" providing enormous SERS enhancement are created inside the ring. Thus, using this structure, SE(R)RS spectra that cannot be acquired directly from Ag colloidal solutions (e.g. $1 \times 10^{-10} \mathrm{M}$ TMPyP by $1 \mathrm{~s}$ accumulation time, phospholipid DSPC) were obtained. Despite the spectral irreproducibility and problems with spurious bands in some cases, SERS microspectroscopy of studied biomolecules using dried Ag colloid/adsorbate systems improves SERS applicability and sensitivity in comparison to measurements directly from Ag colloidal solution. 


\section{Acknowledgments}

The work was supported by grants of the Ministry of Education, Youth and Sports (SVV 265 304) and of the Czech Science Foundation (P208/10/0941).

\section{References}

[1] Surface-Enhanced Raman Scattering: Physics and Applications, K. Kneipp, M. Moskovits, and H. Kneipp, Eds., vol. 103 of Topics in Applied Physics, Springer, Berlin, Germany, 2006.

[2] E. C. Le Ru and P. G. Etchegoin, Principles of Surface-Enhanced Raman Spectroscopy and Related Plasmonic Effects, Elsevier, Amsterdam, The Netherlands, 2009.

[3] C. D. Keating, M. D. Musick, M. H. Keefe, and M. J. Natan, "Kinetics and thermodynamics of Au colloid monolayer self-assembly undergraduate experiments in surface and nanomaterials chemistry," Journal of Chemical Education, vol. 76, no. 7, pp. 949-955, 1999.

[4] P. Šimáková and M. Procházka, "SERRS microspectroscopy of porphyrins on Ag immobilized nanoparticles," Journal of Molecular Structure, vol. 993, no. 1-3, pp. 425-427, 2011.

[5] M. V. Cañamares, J. V. Garcia-Ramos, J. D. Gómez-Varga, C. Domingo, and S. Sanchez-Cortes, "Comparative study of the morphology, aggregation, adherence to glass, and surface-enhanced Raman scattering activity of silver nanoparticles prepared by chemical reduction of $\mathrm{Ag}^{+}$using citrate and hydroxylamine," Langmuir, vol. 21, no. 18, pp. 8546-8553, 2005.

[6] R. D. Deegan, O. Bakajin, T. F. Dupont, G. Huber, S. R. Nagel, and T. A. Witten, "Capillary flow as the cause of ring stains from dried liquid drops," Nature, vol. 389, no. 6653, pp. 827-829, 1997.

[7] E. Kočišová and M. Procházka, "Drop-coating deposition Raman spectroscopy of liposomes," Journal of Raman Spectroscopy, vol. 42, no. 8, pp. 1606-1610, 2011.

[8] N. Leopold and B. Lendl, "A new method for fast preparation of highly surface-enhanced Raman scattering (SERS) active silver colloids at room temperature by reduction of silver nitrate with hydroxylamine hydrochloride," Journal of Physical Chemistry B, vol. 107, no. 24, pp. 5723-5727, 2003.

[9] M. Procházka, B. Vlčková, J. Štěpánek, and P. Y. Turpin, "Probing of porphyrin surface chemistry in systems with laser-ablated Ag nanoparticle hydrosol: role of thiosulfate anions," Langmuir, vol. 21, no. 7, pp. 2956-2962, 2005.

[10] A. E. Aliaga, I. Osorio-Román, P. Leyton et al., "Surface-enhanced Raman scattering study of L-tryptophan," Journal of Raman Spectroscopy, vol. 40, no. 2, pp. 164-169, 2009.

[11] A. Kandakkathara, I. Utkin, and R. Fedosejevs, "Surface-enhanced Raman scattering (SERS) detection of low concentrations of tryptophan amino acid in silver colloid," Applied Spectroscopy, vol. 65, no. 5, pp. 507-513, 2011.

[12] C.-H. Chuang and Y.-T. Chen, "Raman scattering of L-tryptophan enhanced by surface plasmon of silver nanoparticles: vibrational assignment and structural determination," Journal of Raman Spectroscopy, vol. 40, no. 2, pp. 150-156, 2009.

[13] I. W. Levin, "Vibrational spectroscopy of membrane assemblies," in Advances in Infrared and Raman Spectroscopy, R. J. H. Clark and R. E. Heste, Eds., vol. 11, Wiley Heyden, Chichester, UK, 1984. 


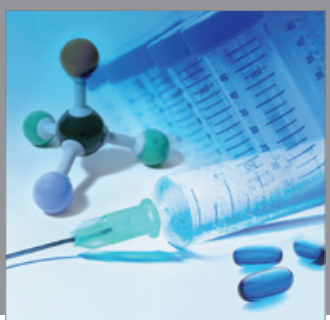

International Journal of

Medicinal Chemistry

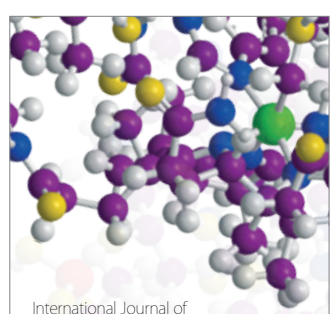

Carbohydrate Chemistry

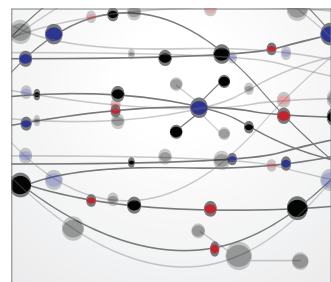

The Scientific World Journal
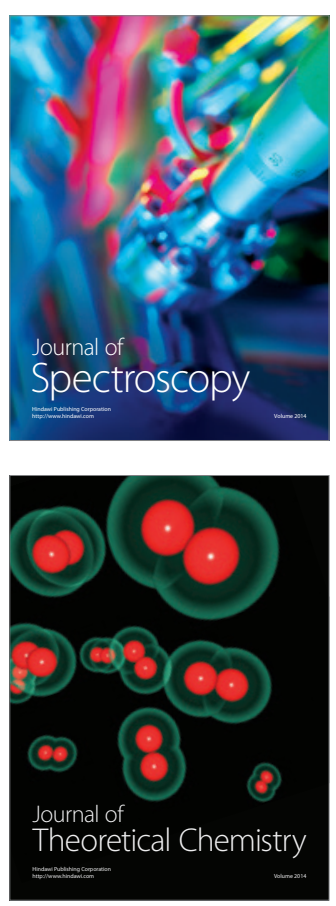
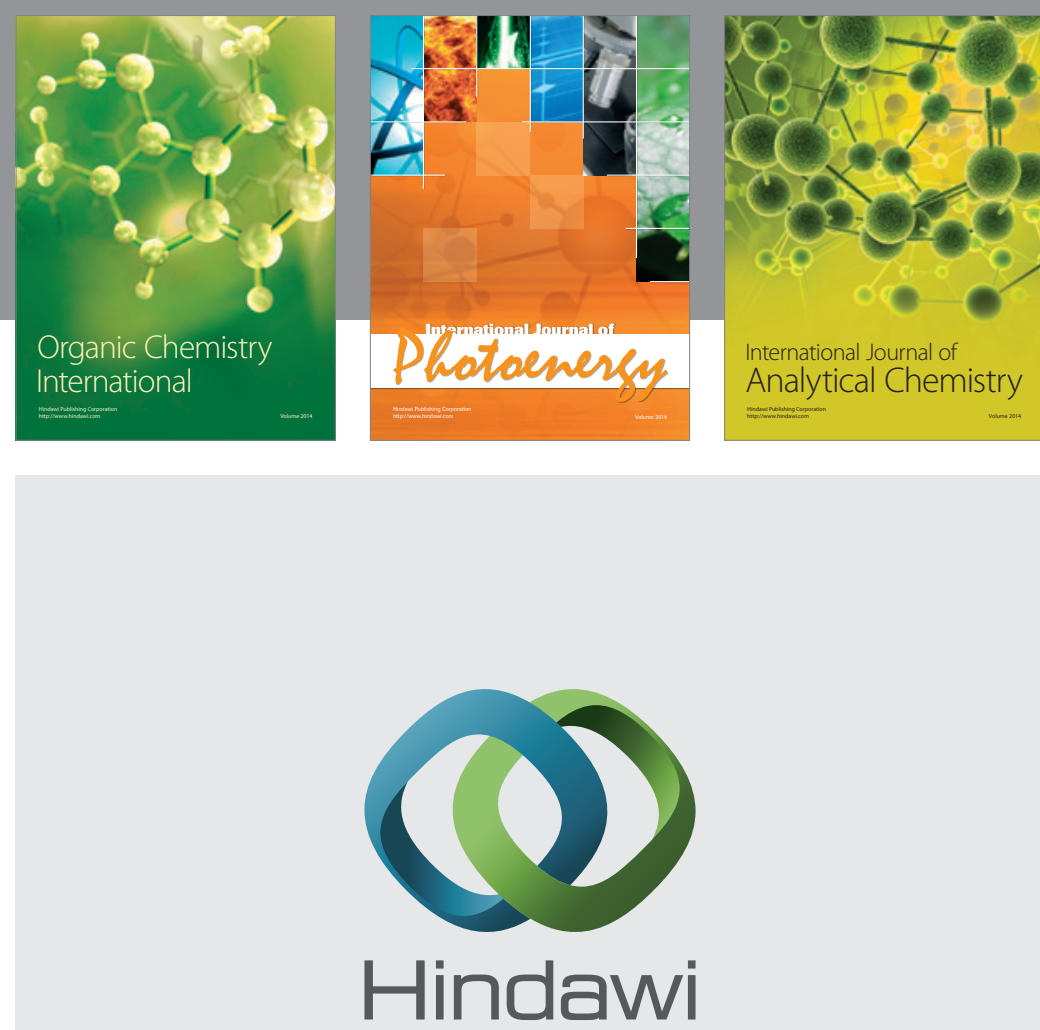

Submit your manuscripts at

http://www.hindawi.com
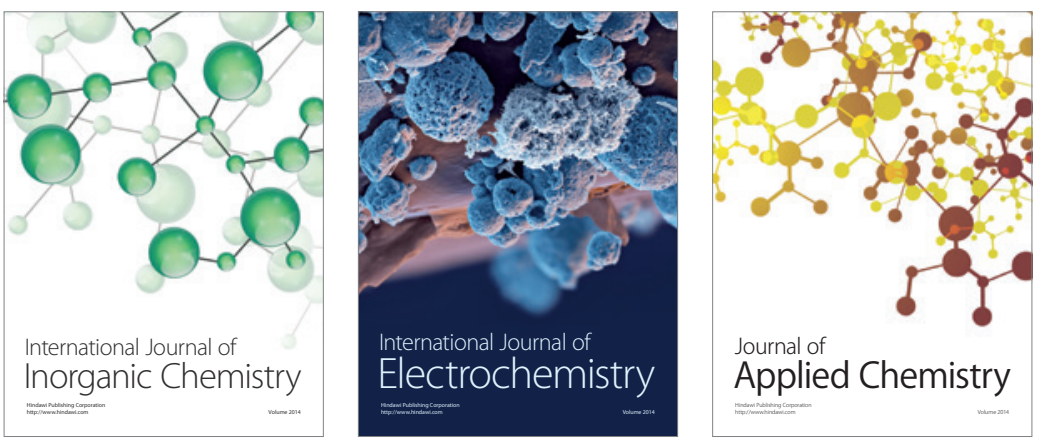

Journal of

Applied Chemistry
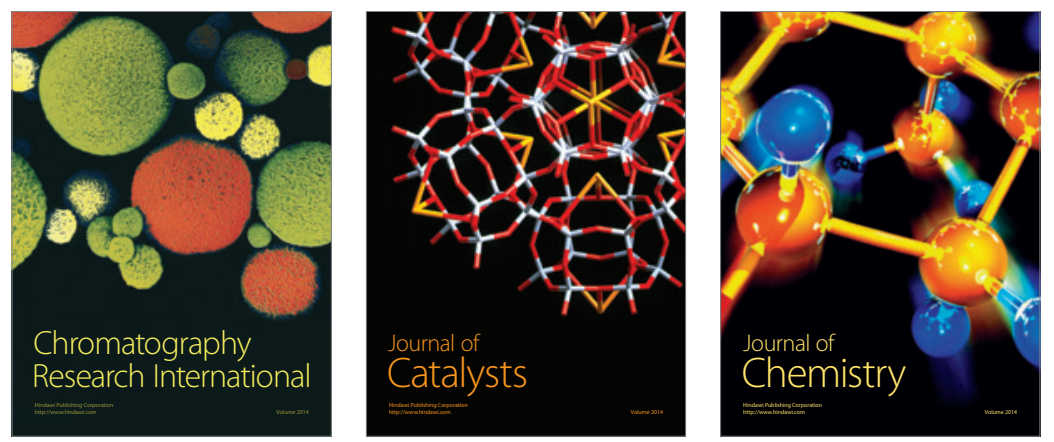
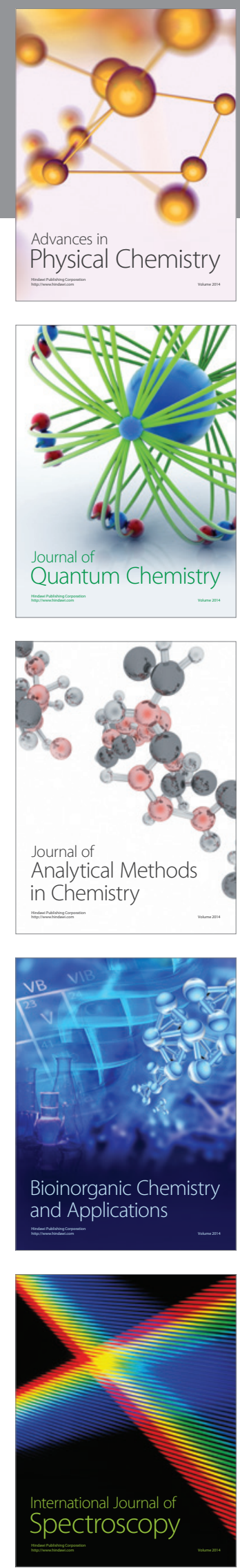